\title{
Neurogenesis in the adult human hippocampus
}

\author{
Peter S. Eriksson ${ }^{1,4}$, Ekaterina Perfilieva ${ }^{1}$, Thomas Björk-Eriksson ${ }^{2}$, Ann-M arie Alborn ${ }^{1}$, \\ Claes Nordborg ${ }^{3}$, Daniel A. Peterson ${ }^{4} \&$ Fred H. Gage ${ }^{4}$ \\ Department of Clinical Neuroscience, Institute of Neurology ${ }^{1}$, Department of Oncology ${ }^{2}$, Department of Pathology ${ }^{3}$, \\ Sahlgrenska University Hospital, 41345 Göteborg, Sweden \\ ${ }^{4}$ Laboratory of Genetics, The Salk Institute for Biological Studies, 10010 North Torrey Pines Road, La Jolla, \\ California 92037, USA \\ Correspondence should be addressed to F.H.G.
}

\begin{abstract}
The genesis of new cells, including neurons, in the adult human brain has not yet been demonstrated. This study was undertaken to investigate whether neurogenesis occurs in the adult human brain, in regions previously identified as neurogenic in adult rodents and monkeys. Human brain tissue was obtained postmortem from patients who had been treated with the thymidine analog, bromodeoxyuridine (BrdU), that labels DNA during the S phase. Using immunofluorescent labeling for BrdU and for one of the neuronal markers, NeuN, calbindin or neuron specific enolase (NSE), we demonstrate that new neurons, as defined by these markers, are generated from dividing progenitor cells in the dentate gyrus of adult humans. Our results further indicate that the human hippocampus retains its ability to generate neurons throughout life.
\end{abstract}

Loss of neurons is thought to be irreversible in the adult human brain, because dying neurons cannot be replaced. This inability to generate replacement cells is thought to be an important cause of neurological disease and impairment. In most brain regions, the generation of neurons is generally confined to a discrete developmental period. Exceptions are found in the dentate gyrus and the subventricular zone of several species that have been shown to generate new neurons well into the postnatal and adult period ${ }^{1-6}$. Granule neurons are generated throughout life from a population of continuously dividing progenitor cells re siding in the subgranular zone of the dentate gyrus in the rodent brain ${ }^{5}$. 'N ewborn' neurons generated from these progenitor cells migrate into the granule cell layer, differentiate, extend axons and express neuronal marker proteins ${ }^{7-10}$

We examined whether progenitor cells reside in the adult human hippocampus and whether new neurons are born within the dentate gyrus of the adult human brain. Postmortem tissue from the hippocampus and the subventricular zone of caudate nucleus was obtained from cancer patients $(n=5)$ who received

Fig. 1 Newly generated cells can be detected in the adult human brain in patients previously treated with BrdU. a, The hippocampal region of the adult human brain immunoperoxidase-stained for the neuronal marker NeuN. b, The hippocampal dentate gyrus granule cell layer (GCL) visualized with immunoperoxidase staining for NeuN. c, Differential interference contrast photomicrograph showing BrdU-labeled nuclei (arrows) in the dentate granule cell layer (GCL). d, Differential interference contrast photomicrograph showing a BrdU-labeled nucleus (arrow) in the human dentate GCL. BrdU-positive nuclei have a rounded appearance and resemble the chromatin structure of mature granule cells and are found within the granule cell layer. e, Differential interference contrast photomicrograph showing BrdUpositive cells (arrows) adjacent to the ependymal lining in the subventricular zone of the human caudate nucleus. Cells with elongated nuclei resembling migrating cells are in the rat subventricular zone (SVZ). f, Differential interference contrast photomicrograph showing BrdU-positive cells (arrows) with round to elongated nuclei in the subventricular zone of the human caudate nucleus. All scale bars represent $50 \mu \mathrm{m}$. one intravenous infusion ( $250 \mathrm{mg} ; 2.5 \mathrm{mg} / \mathrm{ml}, 100 \mathrm{ml}$ ) of bromodeoxyuridine (BrdU) for diagnostic purposes ${ }^{11}$. One patient diagnosed with a similar type and location of cancer, but without BrdU treatment, was included as a control. A thymidine analog, BrdU is incorporated into the DNA of dividing cells and can be detected immunohistochemically in their progeny $y^{5,12,13}$.

Cell genesis and survival in the adult human dentate gyrus The number of surviving labeled, proliferating progenitors was
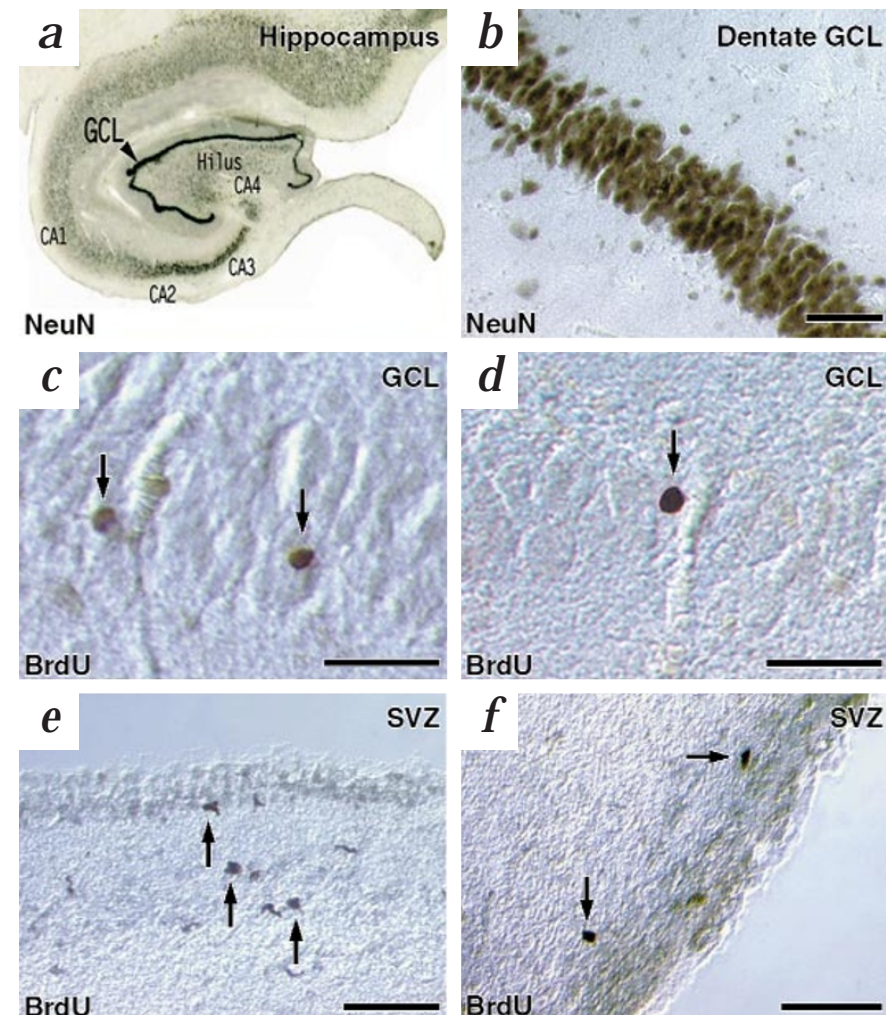
a

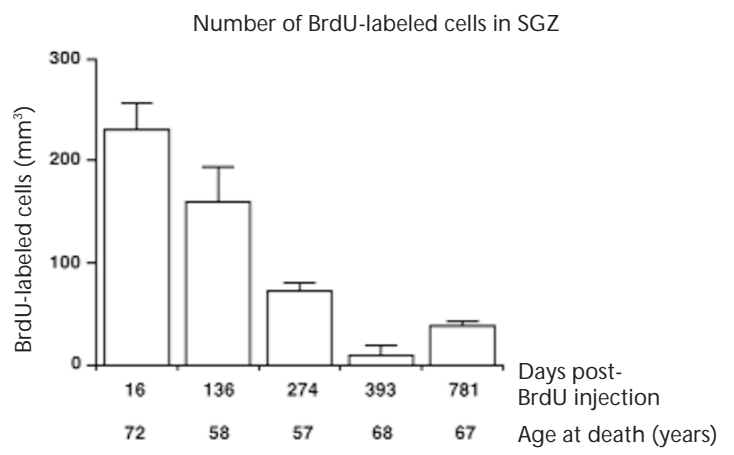

Fig. 2 Quantitation of newly generated cells in the adult human hippocampus. The density of BrdU immunoperoxidase-stained cells in the subgranular zone (SGC) (a) and the granule cell layer (GCL) (b) and the hilus (c) was determined in 5 to 7 sections per patient (mean number of BrdUpositive cells per $\mathrm{mm}^{3}$ sample volume \pm s.e.m.) The corresponding patient's age at the time of death and interval as BrdU infusion are given for each BrdU-treated patient $(n=5)$.

quantified using immunohistochemical staining for BrdU and unbiased counting techniques ${ }^{14,15,16}$. BrdU-labeled cells were quantified in the granule cell layer and the subgranular zone of the dentate gyrus and in the hilus (area CA4; Fig. 1a). In all BrdU-treated patients, the granule cell layer contained BrdU-positive, round- to oval-shaped nuclei with the typical morphology of granule cell neuron nuclei (Fig. 1b-d). The morphological appearance and localization of these nuclei were similar to those of the nuclei of cells found in the dentate gyrus of adult mouse, rat and marmoset monkey,6,17. No BrdU-immunoreactive profiles could be detected in the control patient that had not received BrdU. The presence of BrdU-positive nuclei in a brain region that is neurogenic in many other mammalian and primate species indicates that proliferating neural progenitor cells are present in the adult human dentate gyrus.

The number of BrdU-positive cells in the granule cell layer, the subgranular zone and hilus varied between individuals, as may be expected because of the varying post-infusion intervals and different ages of the patients (Fig. 2a-C). There is an apparent decline in the number of cells that are detected in the patients that had the longest interval between BrdU treatment and histological assessment. This decline may indicate a progressive death of the newly generated cells over time. However, the small sample weakens any quantitative conclusion.

\section{BrdU-labeled cells co-express neuronal markers}

To determine the cell fate of the BrdU-immunoreactive cells, we did triple immunofluorescent labeling for BrdU and cell-specific markers, including glial fibrillary acidic protein (GFAP), a marker for astroglia, and one of the neuronal markers, NeuN ${ }^{18,19}$, calbindin ${ }^{20}$ or neuron specific enolase ${ }^{21}$ (NSE). Confocal microscopy was used to determine the phenotype of the BrdU-positive cells (Figs. 3 and 4). No evidence for cross-reactivity between GFAPimmunoreactivity and any of the three neuronal markers was detected in these sections. GFAP-immunopositive processes were observed to surround neurons and their processes, but did not co-express in the same cell (Fig. 3e-h). Autofluorescence was observed in this tissue, but the nonspecific emission artifact was easily detected by its contribution to multiple wavelengths (Fig. 3a-d). We took care to evaluate potential sources of autofluores- b

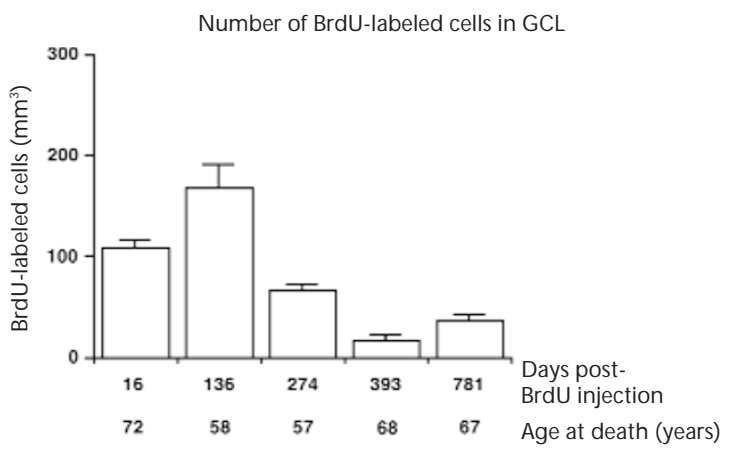

C

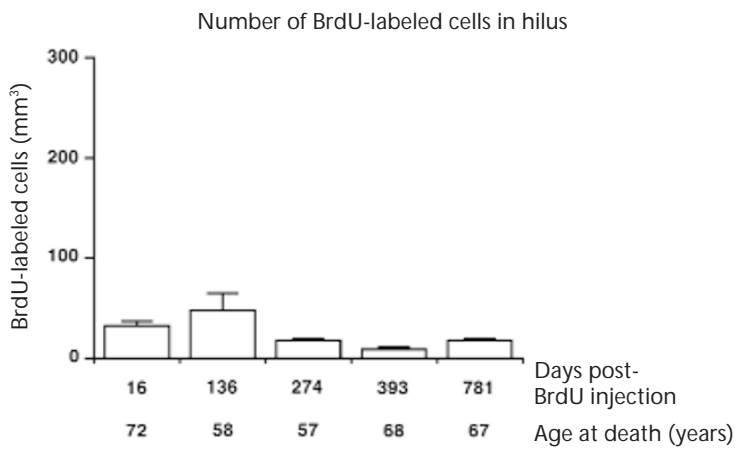

cence in the human tissue by di rectly imaging untreated sections and sections from the control patient both with and without the pretreatment for BrdU-immunohistochemical detection in the absence of antibodies against BrdU. The autofluorescence was present even in completely untreated sections and was restricted to the cytoplasm of neuronally marked cells or to endothelial cells and red blood cells and could be clearly distinguished from BrdU-immunoreactive profiles (Fig. 3). The majority of the NeuN-positive neurons that double-labeled with BrdU were located within or near the granule cell layer and had the morphological characteristics of granule cell neurons: round or oval nuclei with small- to medium-sized cell bodies (Fig. 3). Confocal microscopic Z-series analyses of BrdU-positive nuclei unambiguously identified double-labeling with NeuN and demonstrated the existence of newly generated (BrdU-labeled) granule cell neurons in the dentate gyrus of the adult human brain (Fig. 3d-h). The phenotypic expression of these BrdU-positive neurons in adult humans was equivalent to the expression found in adult rodents $5^{5,10,12,17}$ (Fig. 3i-I). The average fraction of BrdU-NeuN double-labeled cells in the granule cell layer was $22.0 \pm 2.4 \%$ among the BrdU-treated patients.

Neurons double-labeled with BrdU and NSE could also be detected in all BrdU-treated subjects (Fig. 4e-f). The average fraction of BrdU-labeled cells that were also NSE-labeled in the granule cell layer was $22.7 \pm 2.8 \%$. Cells that double-labeled for BrdU and calbindin were also observed and provided further support for the occurrence of neurogenesis in the adult SGZ, GCL and hilus (Fig. 4a-d). The average fraction of BrdU-labeled cells that were also cal bindin-positive in the granule cell layer was 7.9 $\pm 2.2 \%$ among the BrdU-treated patients. The dentate gyrus from all individuals contained a fraction of BrdU-positive cells that were also GFAP-positive, $18.1 \pm 1.8 \%$, and had the typical morphology of star-shaped glial cells with small, irregularly shaped nuclei and small cell bodies with thin, GFAP-positive processes (Fig. 4c-d). The dentate gyrus from all subjects contained BrdU- 
Fig. 3 Newly generated cells in the adult human dentate gyrus can express a neuronal phenotype. Simultaneous detection of immunofluorescent labels for NeuN (a; scale bar represents $25 \mu \mathrm{m}$ ), BrdU (b) and GFAP (c) for detection of astrocytes and a merge of these signals in $x-, y-$ and $z$ registration (d) examined by confocal microscopy showed that BrdU-labeled nuclei could specifically co-express NeuN without expressing GFAP (arrows in a-d). In addition to specific BrdU-labeling, some neurons contained nonspecific fluorescence in the green and red emission, reflecting their accumulation of lipofuchsin granules (arrowheads in a-d). Red blood cells and endothelial cells, present in several small blood vessels, also emit nonspecific green and red fluorescence (small blood vessel indicated by arrowheads, larger blood vessels indicated by asterisks in e-h). The specificity of BrdU-NeuN co-expression in three-dimensions is demonstrated by a series of focal planes above $(\mathbf{e}, \mathbf{f})$ and below $(\mathbf{g}, \mathbf{h})$ the focal plane shown in $d$ (arrows indicate the same cell as in d). The appearance of adult BrdU-positive neurons in the
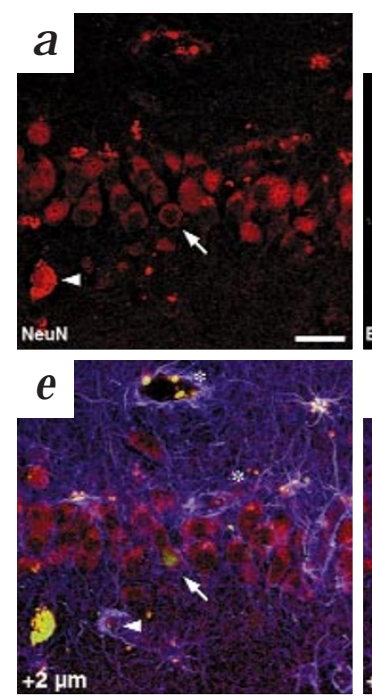
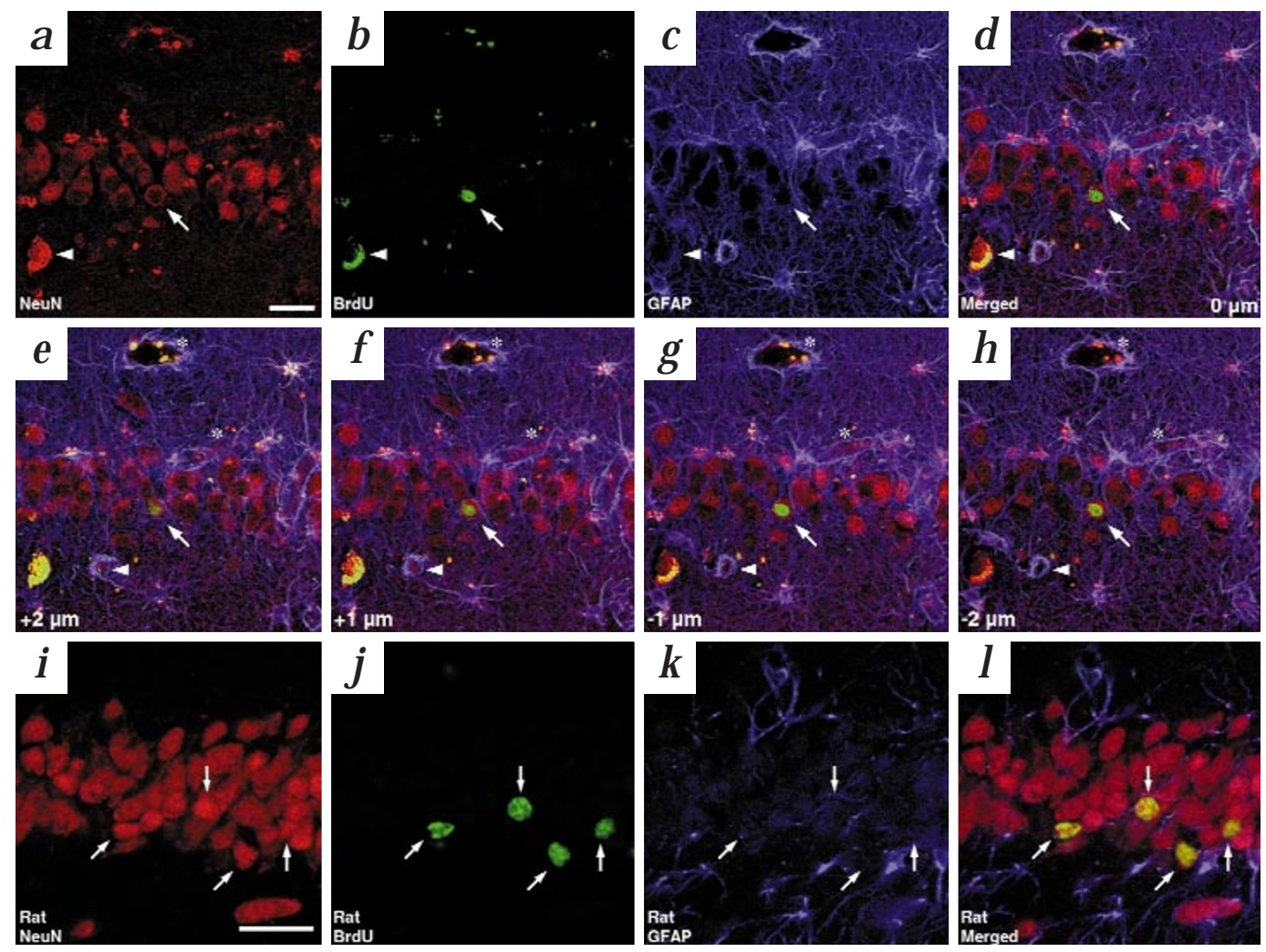

human dentate gyrus is equivalent to BrdU-labeled neurons in the adult rat dentate gyrus (i-I; scale bar represents $25 \mu \mathrm{m}$ ). The rat tissue (i-I) is at a different magnification than the human tissue $(a-h)$. positive cells that were immunonegative for both neuronal and glial markers. These immunonegative cells likely represent quiescent undifferentiated cells, newborn cells of a phenotype not examined here and/or a pool of asymmetrically dividing progenitor cells. These cells were characterized by small, roundto-oval BrdU-positive nuclei and the absence of cell-specific immunoreactivity.

To further confirm the presence of neurogenesis, we double-labeled using antibodies against BrdU and either NSE, calbindin or NeuN with chromagens for brightfield optics (alkaline phosphatase (Vector Blue) for BrdU and 3-amino-9-ethylcarbazole (AEC) for the neuronal markers). Although confocal microscopy could not be used for imaging, the brightfield chromagens had the advantage of not fading with examination and not contributing autofluorescence to the image. Examination of brightfield staining confirmed that BrdU-positive cells in the adult human hippocampus could express a neuronal phenotype (Fig. 5a-e) morphologically indistinguishable from adult rodent BrdU-positive neurons (Fig. $5 f$ and g), strongly supporting our conclusion that neurogenesis occurs in the dentate granule cell layer of the adult human brain.

\section{BrdU labeling in the subventricular zone of adult human brain}

Another neurogenic region, the subventricular zone (SVZ) adjacent to the caudate nucleus, was examined for the presence of BrdU-positive cells. Tissue samples from all BrdU-treated patients contained BrdU-positive cells within the SVZ (Fig. le-f). BrdU-positive cells did not co-express the cell-specific markers GFAP and NeuN (data not shown). The morphology of BrdU-labeled nuclei within the SVZ was small and round-to-oval, resem- bling that of progenitor cells in the rat SVZ (ref. 5). This finding supports the idea that the human SVZ contains progenitor cells and that these cells are required to migrate from the SVZ before they differentiate ${ }^{22}$. We were unable to detect any BrdU-immunoreactive cells in tissue from the control patient who had not received BrdU treatment, supporting the interpretation that the BrdU staining we report here reflects the persistence in the adult human brain of cell genesis.

\section{Discussion}

Our study demonstrates that cell genesis occurs in human brains and that the human brain retains the potential for self-renewal throughout life. Although earlier studies in adult primates have been unsuccessful in showing neurogenesis in the dentate gyrus ${ }^{23,24}$, a recent report has demonstrated neurogenesis in three-year-old marmoset monkeys ${ }^{6}$. Although the number of BrdU-labeled cells entering the neuronal lineage seems to be lower in the human hippocampus than in marmosets, those monkeys ${ }^{6}$ were considerably younger, even in relative terms, than the humans examined here (average age of $64.4 \pm 2.9$ years). Therefore, we conclude that, as in rodents $5^{5,25}$, neurogenesis in the human dentate gyrus continues throughout life.

Although our results demonstrate that cells in the adult brain undergo cell division and that some of the newly generated cells can survive and differentiate into cells with morphological and phenotypic characteristics of neurons, we have not proven that these newly generated cells are functional. We also do not yet know the biological significance of cell genesis in the adult human brain. However, this does provide a basis to investigate a newly discovered type of 'neuroplasticity' in humans, one based 
Fig. 4 Newly generated cells in the adult human dentate gyrus can express additional neuronal phenotypes. The calcium-binding protein, calbindin, is expressed by certain neuronal populations, in-
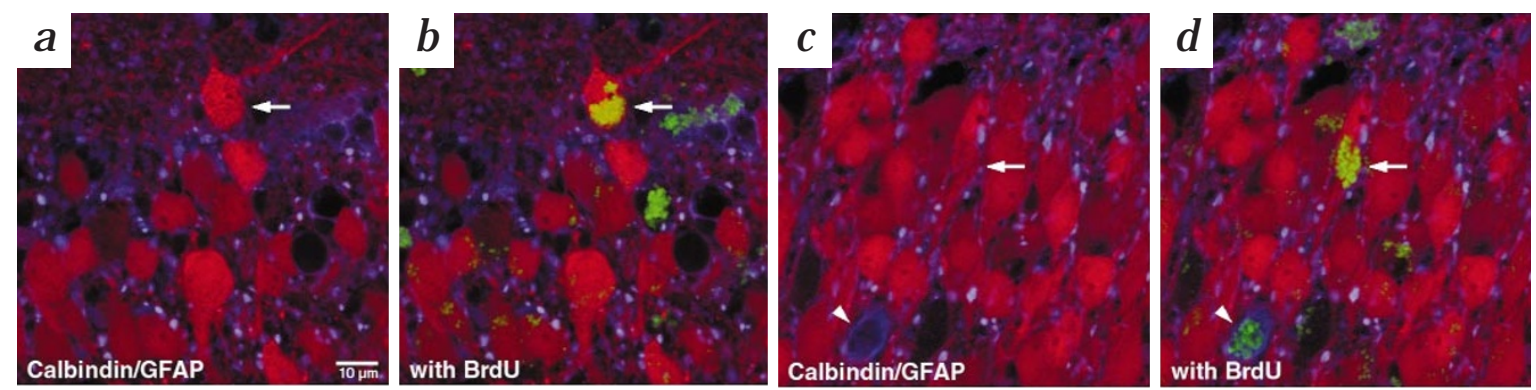

cluding dentate granule neurons, in vivo. a, Fluorescent labeling of calbindin (red) and GFAP (blue) discriminated between granule neurons and astrocytes. Scale bar represents $10 \mu \mathrm{m}$. The arrow indicates a newly generated, BrdU-labeled calbindinpositive neuron shown with the label-colors merged in b. c, Another calbindin-positive neuron (arrow) that co-expresses BrdU (d). Newly generated cells may also differentiate into astrocytes (c,d; arrowheads). BrdU-labeled cells can also express neuron specific enolase (NSE, shown in red; arrows in $\mathbf{e}$ and $\mathbf{f}$ ) without expressing GFAP (blue).

on addition of neurons, that has not been previously considered. Studies in rodents have shown that the adult hippocampus contains progenitor cells that can be expanded in vitro and grafted back into the adult brain, where they can respond to regional cues by differentiation into site-specific phenotypes, including neurons $s^{26,27}$. The presence of progenitor cells in the human dentate gyrus, reported here, indicates that these cells also may be used for in vitro and in vivo studies of cell differentiation and possibly subsequent transplantation studies. Furthermore, environmental stimulation can influence the rate of neurogenesis in the adult and senescent rodent dentate gyrus ${ }^{12,17}$. The potential to regulate human neurogenesis should prove to be an interesting area of investigation.

\section{Methods}

Autopsy material. Human hippocampal tissue was obtained at autopsy with the full consent of each family. All patients were diagnosed with squamous cell carcinomas at the base of the tongue, in the larynx or in the pharynx. All patients received bromodeoxyuridine (BrdU) $(250 \mathrm{mg})$ dissolved in
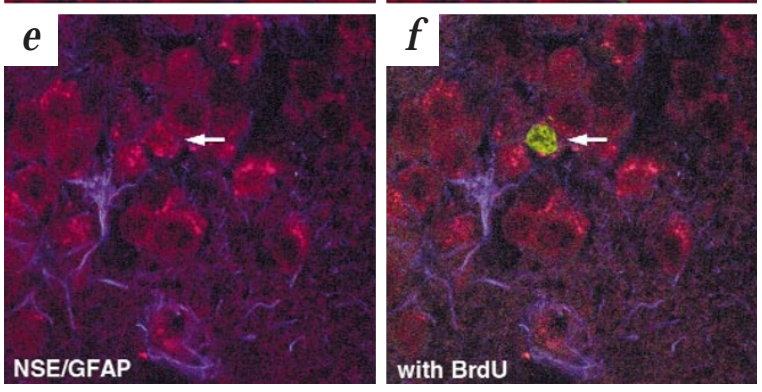

saline and given as an intravenous infusion $(2.5 \mathrm{mg} / \mathrm{ml}, 100 \mathrm{ml})$. The BrdU was given to the patients to assess the proliferative activity of the tumor cells, expressed as BrdU-labeling index. No signs of macroscopic or microscopic metastases were found in autopsy material from the cerebrum in any of the patients. No anti-cancer therapy was administered before or during BrdU administration to any of the patients.

Tissue preparation. The hippocampal formation and ventricular zone were dissected out and post-fixed in $4 \%$ paraformaldehyde for 24 hours and then transferred into $30 \%$ sucrose solution until equilibrated. The hippocampi were sectioned (slices $40 \mu \mathrm{m}$ in thickness) in the coronal plane on a sliding microtome and stored at $-20{ }^{\circ} \mathrm{C}$ in a cryoprotecting buffer containing $25 \%$ ethylene glycol, $25 \%$ glycerin and $0.05 \mathrm{M}$ phosphate buffer. For comparison, sections derived from BrdU-injected adult rats and mice that had been intracardially perfused with $4 \%$ paraformaldehyde were processed in parallel with the human tissue.

Histology. Immunohistochemical detection of BrdU requires a pre-treatment of the sections to denature DNA (ref. 5). All staining was done on free-floating sections and the blocking buffer contained both 3\% normal donkey serum and 3\% normal human serum (Sigma). For sections stained only for BrdU, a
Fig. 5 Brightfield double-immunohistochemical demonstration of neuronal phenotype. a, Staining with the neuronal marker NeuN labels both dentate granule cells (top inset, shown in b) and hilar neurons (bottom inset,
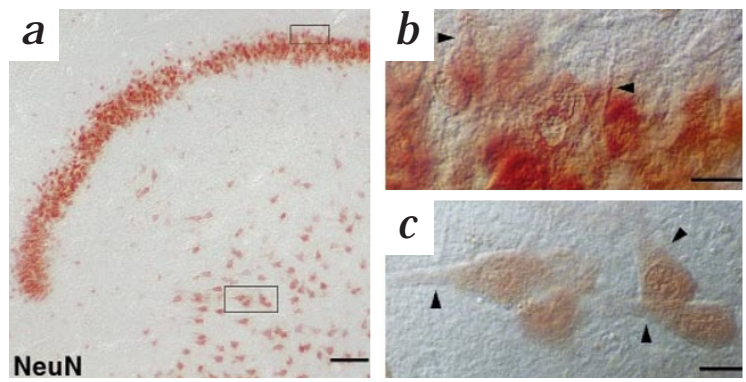

shown in c). Scale bar represents $100 \mu \mathrm{m}$. Combining NeuN staining with differential interference contrast optics demonstrated that NeuN labeling included the entire nucleus and perikaryal cytoplasm and extended into proximal portions of major dendrites (arrowheads) in both granule cells (b; scale bar represents $20 \mu \mathrm{m}$ ) and hilar neurons (c, scale bar represents $20 \mu \mathrm{m}$ ). Newly generated cells could be found in the dentate granule layer when detected with antibodies against BrdU in conjunction with NeuN staining (d, scale bar represents $25 \mu \mathrm{m}$ ). Dark blue-stained BrdU-labeled nuclei can co-express the neuronal marker NeuN shown in red (e, scale bar represents $10 \mu \mathrm{m})$. The appearance of BrdU-labeled cells in adult human dentate gyrus is equivalent to that of the adult rat dentate gyrus (f, scale bar represents $25 \mu \mathrm{m}$; and $\mathbf{g}$, scale bar represents $10 \mu \mathrm{m}$.).
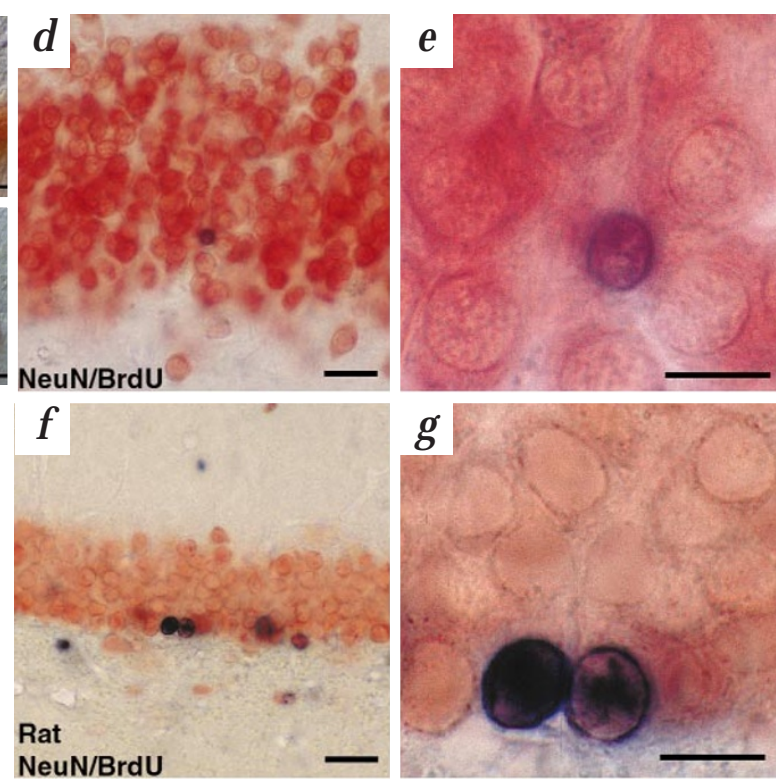
mouse-anti-BrdU antibody (Boehringer; diluted 1:400) was used in combination with the avidin-biotin complex method using a biotinylated donkey antimouse-IgG antibody (Vector Laboratories, Burlingame, California; diluted 1:167) and reacted with a diaminobenzidine (DAB) chromagen.

Immunofluorescent double- and triple-labeling was done as described $^{5,12}$. For multiple immunostaining, BrdU was detected with a rat antiBrdU antibody (Harlan Sera-Lab, Loughborough, England; diluted 1:500) and visualized with a FITC-conjugated secondary donkey anti-rat antibody (Jackson ImmunoResearch, West Grove, Pennsylvania; diluted 1:250). For neuronal phenotype markers, sections were incubated with one of the following antisera: rabbit anti-calbindin antiserum (SWant, Bellinzona, Switzerland; diluted 1:1,000), or mouse anti-NeuN antiserum (from R. Mullen $^{18}$; diluted 1:20), or rabbit anti-NSE antiserum (Polysciences, Warrington, Pennsylvania; diluted 1:800). These neuronal markers were visualized by using the species-appropriate Cy3-conjugated secondary antibody (Jackson ImmunoResearch, West Grove, Pennsylvania; diluted 1:250). GFAP was detected in the same sections using a guinea pig anti-GFAP antiserum (Advanced Immunochemicals, Long Beach, California; diluted 1:250) and visualized using a Cy5-conjugated donkey anti-guinea pig antibody (Jackson ImmunoResearch, West Grove, Pennsylvania; diluted1:250).

For double immunostaining using brightfield chromagens, sections were incubated with a pooled solution of rat anti-BrdU (Harlan Sera-Lab, Loughborough, England; diluted 1:500) and mouse anti-NeuN (from R. Mullen $^{18}$; diluted 1:100) antisera. After being rinsed and blocked, sections were incubated first with a biotinylated donkey anti-rat antibody (Jackson ImmunoResearch, West Grove, Pennsylvania; diluted 1:250), followed by incubation with an alkaline phosphatase avidin-biotin substrate and then reaction with the blue chromagen (Vector Blue; Vector Laboratories, Burlingame, California). After being rinsed and blocked further, the sections then were incubated with a biotinylated donkey anti-mouse antibody (Jackson ImmunoResearch, West Grove, Pennsylvania; diluted 1:250) followed by incubation with a peroxidase avidin-biotin substrate and then reaction with the red chromagen (3-amino-9-ethylcarbazole or AEC; Vector Laboratories, Burlingame, California). Sections were mounted and coverslipped with an aqueous mounting medium.

Fluorescent signals were imaged using a confocal laser scanning microscope equipped with a krypton/argon mixed gas laser (Bio-Rad MRC1024; Bio-Rad, Richmond, California) using individual collection of wavelengths to minimize artifactual detection of non-specific fluorescent emission. At least 20 BrdU-positive cells within multiple immunofluorescently stained sections from each patient were examined on the confocal microscope to determine the phenotype of BrdU-labeled cells. Brightfield images were derived from conventional photomicrographs digitized by using a slide scanner. Figures were composed by using Adobe Photoshop (Adobe Systems, Mountain View, California) with image adjustments confined to creating an equivalent signal distribution between panels.

Quantitation. The numbers of BrdU-positive cells in the granule cell layer, the subgranular layer and the hilus, and their corresponding sample volumes were determined in five to seven immunoperoxidase-stained coronal sections $40 \mu \mathrm{m}$ in thickness, at least $240 \mu \mathrm{m}$ apart, from each patient. Area estimations were done on an IBAS image analysis system. The section thickness of $40 \mu \mathrm{m}$ (microtome setting) was used in the dissector estimation of volume. The number of BrdU-positive cells was counted within the granule cell layer (GCL) and two cell diameters below the granule cell layer, ignoring the cells in the uppermost focal plane and focusing through the thickness of the section (optical disector principle; refs. 14,15,16) to avoid oversampling errors. The subgranular zone (SGZ) was defined as the area from one cell diameter within the GCL from the hilus-GCL border and two cell diameters below the hilus-GCL border (Fig. 1). Quantitative analysis of BrdU immunoperoxidase-stained sections was done on a Nikon microscope equipped with a video camera. The results are expressed as BrdU-positive cells per sample volume per section.

\section{Acknowledgments}

We thank G. Kempermann, T. Palmer, E. Brandon, M.-C. Senut, K. Sakurada, L. Chehabeddine and M. L. Gage for their comments, and H. van Praag for the contribution of rat tissue. In addition, we thank L. Kitabayashi for technical assistance. This study was supported by grants from the Swedish M edical
Research Council (project no. K98-12X-12535-01A), Faculty of M edicine, University of Göteborg, the Gunvor and Josef Anérs Stiftelse, the John and Brit Wennerströms Stiftelse for N eurologisk Forskning, the Rune and Ulla Amlövs Stiftelse for N eurologisk and Reumatologisk Forskning, N HR-fonden, Stiftelsen Göteborgs MS förenings forsknings och byggnadsfond, Stiftelsen Handlanden Hjalmar Svenssons Forskningsfond, Göteborgs Läkaresällskap, Hjärnfonden, The Swedish Society of M edicine, Stiftelsen Lars Hiertas M inne, Stiftelsen Assar Gabrielssons Fond and the Edit Jacobssons Fond and from NIA and NINDS and the Alzheimer's Association (F.H.G.) and the American Federation for Aging Research (D.A.P.).

\section{RECEIVED 9 SEPTEMBER; ACCEPTED 13 OCTOBER 1998}

1. Altman, J. \&. Das, G.D. Autoradiographic and histological evidence of postnatal hippocampal neurogenesis in rats. J. Comp. Neurol. 124, 319-335 (1965).

2. Altman, J. \& Das, G.D. Postnatal neurogenesis in the guinea-pig. Nature 214, 1098-1101 (1967).

3. Caviness, V.S. Time of neuron origin in the hippocampus and dentate gyrus of normal and reeler mutant mice: an autoradiographic analysis. J. Comp. Neurol. 151, 113-120 (1973)

4. Gueneau, G., Privat, A., Drouet, J. \& Court, L. Subgranular zone of the dentate gyrus of young rabbits as a secondary matrix. A high-resolution autoradiographic study. Dev. Neurosci. 5, 345-358 (1982).

5. Kuhn, H.G., Dickinson-Anson, H. \& Gage, F.H. Neurogenesis in the dentate gyrus of the adult rat: Age-related decrease of neuronal progenitor proliferation. J. Neurosci. 16, 2027-2033 (1996).

6. Gould, E., Tanapat, P., McEwen, B.S., Flugge, G. \& Fuchs, E. Proliferation of granule cell precursors in the dentate gyrus of adult monkeys is diminished by stress. Proc. Natl. Acad. Sci. USA 95, 3168-3171 (1998).

7. Kaplan, M.S. \& Bell, D.H. Mitotic neuroblasts in the 9-day-old and 11-month-old rodent hippocampus. J. Neurosci. 4, 1429-1441 (1984).

8. Kaplan, M.S. \& Hinds, J.W. Neurogenesis in the adult rat: electron microscopic analysis of light radioautographs. Science 197, 1092-1094 (1977).

9. Stanfield, B.B. \& Trice, J.E. Evidence that granule cells generated in the dentate gyrus of adult rats extend axonal projections. Exp. Brain Res. 72, 399-406 (1988).

10. Cameron, H.A., Woolley, C.S., McEwen, B.S. \& Gould, E. Differentiation of newly born neurons and glia in the dentate gyrus of the adult rat. Neuroscience 56, 337-344 (1993)

11. Dolbeare, F. Bromodeoxyuridine: a diagnostic tool in biology and medicine, Part I: Historical perspectives, histochemical methods and cell kinetics. Histochem. J. 27, 339-369 (1995)

12. Kempermann, G., Kuhn, H.G. \& Gage, F.H. Genetic influence on neurogenesis in the dentate gyrus of adult mice. Proc. Natl. Acad. Sci. USA 94, 10409-10414 (1997).

13. del Rio, J.A. \& Soriano, E. Immunocytochemical detection of 5'-bromodeoxyuridine incorporation in the central nervous system of the mouse. Dev. Brain Res. 49, 311-317 (1989).

14. Gundersen, H.J. et al. The new stereological tools: disector, fractionator, nucleator and point sampled intercepts and their use in pathological research and diagnosis. APMIS 96, 857-881 (1988)

15. West, M.J. Regionally specific loss of neurons in the aging human hippocampus. Neurobiol. Aging 14, 287-293 (1993).

16. Coggeshall, R.E. \& Lekan, H.A. Methods for determining numbers of cells and synapses: a case for more uniform standards of review. J. Comp. N eurol. 364, 6-15 (1996).

17. Kempermann, G., Kuhn, H.G. \& Gage, F.H. More hippocampal neurons in adult mice living in an enriched environmen Nature 386, 493-495 (1997).

18. Mullen, R.J., Buck, C.R. \& Smith, A.M. NeuN, a neuronal specific nuclear protein in vertebrates. Development 116, 201-211 (1992).

19. Wolf, H.K. et al. NeuN: A useful neuronal marker for diagnostic histopathology. J. Histochem. Cytochem. 44, 1167-1171 (1996).

20 Sloviter, R.S. Calcium-binding protein (calbindin-D28k) and parvalbumin immunocytochemistry: localization in the rat hippocampus with specific reference to the selective vulnerability of hippocampal neurons to seizure activity. J. Comp. Neurol. 280, 183-196 (1989).

21. Schmechel, D., Marango, P.J., Zis, A.P., Brightman, M. \& Goodwin, F.K. Brain enolases as specific markers of neuronal and glial cells. Science 199, 313-315 (1978).

22. Kirschenbaum, B. et al. In vitro neuronal production and differentiation by precursor cells derived from the adult human forebrain. Cereb. Cortex 4, 576-589 (1994).

23. Rakic, P. Limits of neurogenesis in primates. Science 227, 1054-1056 (1985).

24. Eckenhoff M.F. \& Rakic, P. Nature and fate of proliferative cells in the hippocampal dentate gyrus during the life span of the rhesus monkey. J. N eurosci. 8, 2729-2747 (1988).

25. Kempermann, G., Kuhn, H.G. \& Gage, F.H. Experience-induced neurogenesis in the senescent dentate gyrus. J. N eurosci. 18, 3206-3212 (1998).

26. Gage, F.H. et al. Survival and differentiation of adult neuronal progenitor cells trans planted to the adult brain. Proc. Natl. Acad. Sci. USA 92, 11879-11883 (1995).

27. Suhonen, J.O., Peterson, D.A., Ray, J. \& Gage, F.H. Differentiation of adult hippocampus-derived progenitors into olfactory neurons in vivo. N ature 383, 624-627 (1996). 